\title{
Quantization of Time-dependent Non-central Singular Potential Systems in Three Dimensions by Using the Nikiforov-Uvarov Method
}

\author{
Salah Menouar \\ Laboratory of Optoelectronics and Compounds (LOC), Departement of Physics, \\ Faculty of Science, University of Ferhat Abbas Setif 1, Setif 19000, Algeria \\ Jeong Ryeol CHOI* \\ Department of Radiologic Technology, Daegu Health College, Daegu 41453, Korea
}

(Received 19 October 2015, in final form 6 January 2016)

\begin{abstract}
Quantum solutions of a time-dependent Hamiltonian for the motion of a time-varying mass subjected to time-dependent singular potentials in three dimensions are investigated. A time-dependent inverse quadratic potential and a Coulomb-like potential are considered as the components of the singular potential of the system. Because the Hamiltonian is a function of time, special techniques for deriving quantum solutions of the system are necessary. A quadratic invariant operator is introduced, and its eigenstates are derived using the Nikiforov-Uvarov method together with a unitary transformation method. The Nikiforov-Uvarov method enables us to solve the eigenvalue equations of the invariant operator, which are second-order linear diffierential equations, by reducing the original equation to a hypergeometric type. According to the invariant operator theory, the wave functions of the system are represented in terms of the eigenstates obtained in such a way. The difference of the wave functions from the eigenstates of the invariant operator is that the wave functions have time-dependent phases while the eigenstates do not. By determining the phases of the wave functions via the help of the Schrödinger equation, we identify the full wave functions of the system and address their physical implications.
\end{abstract}

PACS numbers: 03.65.Ca, 03.65.Ge, 03.65.Fd

Keywords: Non-central potential, Singular potential, Nikiforov-Uvarov method, Invariant operator, Unitary operator, Wave function

DOI: $10.3938 /$ jkps.68.505

\section{INTRODUCTION}

If the Hamiltonian of a system involves time functions such as a time-dependent mass and/or frequency, we call the system a time-dependent Hamiltonian system (TDHS). To derive analytical quantum solutions of such a system, a rigorous mathematical procedure is necessary. The quantum problem of time-dependent harmonic oscillators that belong to TDHSs has attracted great concern in the physics community after a seminal report by Lewis [1], which revealed the possibility that the analytical quantum solutions were derivable if we knew a classical solution of the given system.

The thermal states of time-dependent harmonic oscillators with and without a singularity are investigated in the literature [2-4]. Quantum properties of a timedependent Morse oscillator that is useful for analyzing specific growth patterns in crystallization processes and biological growth are studied with emphasis on their co- herent states [5]. A Lie algebraic formulation of timedependent harmonic oscillators is fulfilled, and its consequences are applied to particular cases such as quantizing charged particles in time-varying magnetic fields and investigating the evolution of the cosmological constant in cosmology [6-8].

On the other hand, other types of TDHSs beyond time-dependent harmonic oscillators have not been actively studied yet. In a previous work [9], we investigated quantum solutions of the one-dimensional problem of a linearly increasing mass system subjected to a timedependent inverse quadratic potential and the Coulomb potential. Three-dimensional problems of quantum solutions for a non-central potential system with a timedependent mass will be studied in this work. As singular potentials, we will take a time-varying inverse quadratic potential and the Coulomb potential. Our study for a time-dependent non-central potential will be extended to a complicated system that involves time-dependent ring-shaped potentials.

Recently, active research has been executed for both

\footnotetext{
*E-mail: choiardor@hanmail.net
} 
the classical and the quantum characteristics of noncentral potential systems. They are the properties of short-range interactions between distorted nuclei $[10,11]$, ring-shaped molecular potential systems [12], and electrons that are bound in a Coulomb-like potential under the influence of the Aharonov-Bohm effect $[13,14]$. However, most of them belong to stationary systems that do not involve time-dependent parameters in their description.

We will use the invariant operator method which is a good technique when we derive quantum solutions of TDHSs. A quadratic invariant operator of the system will be introduced, and it will be transformed to a simple form by means of the unitary transformation method. Quantum solutions in the transformed system will be derived using the Nikiforov-Uvarov (NU) method [9,1517]. This method is a mathematical manipulation for solving second-order linear diffierential equations by reducing the original equation to a hypergeometric type. Many classes of second-order linear differential equations associated with non-relativistic Schrödinger equations or the eigenvalue equations of an invariant operator can be solved exactly by using the NU method. The quantum solutions evaluated in such a way in the transformed system will be inversely transformed to those of the original system. Based on this procedure, full wave functions in the original system will be obtained.

The organizing of this paper is as follows: The Hamiltonian of the variable mass system subjected to singular potentials will be introduced and the corresponding quadratic invariant operator will be constructed in section II. The eigenvalue equation of the invariant operator is divided into two parts. One is the radial equation of motion and the other is the polar angle equation. Eigenstates of the radial part will be derived in section III. The eigenvalue equation of the angular part for a double ringshaped non-central potential will be evaluated in section IV, and the corresponding full wave functions will be derived in section $\mathrm{V}$. Concluding remarks are given in the last section.

\section{HAMILTONIAN AND INVARIANT OPERATOR}

Some dynamical phenomena, such as transport phenomena in crystals [18], motion of electrons in a plasma [19], chirped tidal gravitational waves [20], and so on, are described in terms of a time-dependent effective mass. In this case, the effective mass can be represented as $\mu(t)=\mu_{0} / g(t)$, where $\mu_{0}$ is a constant and $g(t)$ is a time function [20]. The case of time-decaying mass systems that correspond to increasing $g(t)$ has attracted special interest in the literature [21-27]. We consider a simple solvable case of a decaying particle where $g(t)=1+k t$, which is confined in a time-dependent Coulomb-like potential coupled to an inverse quadratic potential in three dimensions. More precisely speaking, the Hamiltonian of the system we consider is given by

$$
\begin{array}{r}
H(t)=\frac{1+k t}{2 \mu_{0}}\left(p^{2}+\frac{f(\theta)}{r^{2}}\right)-\frac{Z_{0}(1+k t)}{1-k t-\frac{1}{2} k^{2} t^{2}} \frac{1}{r}, \\
r \geq 0,
\end{array}
$$

where $\mu_{0}, k$, and $Z_{0}$ are constants. The time-varying factor in front of $1 / r$ is somewhat complicated as you can see, and you can refer to Appendix A in order to know why we selected this factor. Notice that the bound solutions of the Schrödinger equation for this Hamiltonian exist only when the coefficient of $1 / r$ is negative; Hence, let us assume that $\frac{Z_{0}(1+k t)}{1-k t-k^{2} t^{2} / 2}>0$. According to the choice of $f(\theta)$, the system becomes various kinds of $\theta$ dependent systems that are subjected to particular noncentral potentials, such as a simple ring-shaped potential [28], a Makarov potential [29], and a Berkdemir potential [30], which are necessary in particular for managing quantum theories of molecular systems. We also assume that $f(\theta)$ is a slowly varying function of the angle $\theta$; this assumption enables us to derive the invariant operator that plays a crucial role for developing the quantum theory of the system [see Eq. (3)].

We are interested in deriving solutions of Schrödinger equation of the form

$$
i \hbar \frac{\partial}{\partial t} \psi_{n}(t)=H(t) \psi_{n}(t)
$$

Because the Hamiltonian depends on time, obtaining such solutions by using only a naive separation of variables method may be not an easy task. Hence, we should seek another method useful for this case. The invariant operator method is known to be very useful for solving the Schrödinger equation of a TDHS in a case like this.

From the equation for the invariant operator $I$, which is represented in the form $d I / d t=\partial I / \partial t+[I, H] /(i \hbar)=$ 0 , an invariant operator may be derived. Hence, through a little algebra under the assumption that $f(\theta)$ varies sufficiently slowly with $\theta$ after substituting Eq. (1) in this equation, we have

$$
\begin{aligned}
I(t)= & \frac{1}{2 \mu_{0}}\left(1-k t-\frac{1}{2} k^{2} t^{2}\right)^{2}\left(p^{2}+\frac{f(\theta)}{r^{2}}\right) \\
& +\frac{1}{2} \mu_{0} k^{2} r^{2}+\frac{k}{2}\left(1-k t-\frac{1}{2} k^{2} t^{2}\right)(r p+p r) \\
& -Z_{0}\left(1-k t-\frac{1}{2} k^{2} t^{2}\right) \frac{1}{r} .
\end{aligned}
$$

If we put the eigenvalue equation of $I$ in the form

$$
I \phi_{n}(r, t)=E_{n} \phi_{n}(r, t)
$$

the wave functions of the system are represented in terms of the eigenstates $\phi_{n}(r, t)$. This is the reason why the invariant operator method is useful for deriving quantum solutions of a TDHS. 
Because $I$ is a function of time, the derivation of its eigenstates may be somewhat difficult. To overcome this, we perform a unitary transformation by introducing a unitary operator $U$ of the form

$$
\begin{aligned}
U(t) & =\exp \left[\frac{i \mu_{0} k}{2 \hbar}\left(1-k t-\frac{1}{2} k^{2} t^{2}\right) r^{2}\right] \\
& \times \exp \left[\frac{i}{2 \hbar} \ln \left(1-k t-\frac{1}{2} k^{2} t^{2}\right)(r p+p r)\right]
\end{aligned}
$$

Then, the eigenstates $\phi_{n}(r, t)$ can be transformed by $U$ as

$$
\Phi_{n}(r)=U(t) \phi_{n}(r, t)
$$

and, as a consequence, the eigenvalue equation may be written as

$$
U I U^{-1} \Phi_{n}(r, \theta)=E_{n} \Phi_{n}(r, \theta) .
$$

Through a little algebra, Eq. (7) becomes

$$
\left(\frac{1}{2 \mu_{0}}\left[p^{2}+\frac{f(\theta)}{r^{2}}\right]-\frac{Z_{0}}{r}\right) \Phi_{n}(r, \theta)=E_{n} \Phi_{n}(r, \theta)
$$

As one can see, this is independent of time. In 3dimensional space, this can be rewritten as

$$
\begin{array}{r}
\frac{-\hbar^{2}}{2 \mu_{0}}\left[\frac{1}{r^{2}} \frac{\partial}{\partial r}\left(r^{2} \frac{\partial}{\partial r}\right)+\frac{1}{r^{2} \sin \theta} \frac{\partial}{\partial \theta}\left(\sin \theta \frac{\partial}{\partial \theta}\right)+\frac{1}{r^{2} \sin ^{2} \theta} \frac{\partial^{2}}{\partial \varphi^{2}}\right] \Phi_{n m l}(r, \theta, \varphi)+\left(\frac{f(\theta)}{2 \mu_{0} r^{2}}-\frac{Z_{0}}{r}\right) \Phi_{n m l}(r, \theta, \varphi) \\
=E_{n m l} \Phi_{n m l}(r, \theta, \varphi)
\end{array}
$$

where the eigenfunctions are given in the form

$$
\begin{aligned}
\Phi_{n m l}(r, \theta, \varphi)=R_{n l}(r) \Theta_{n m l}(\theta) e^{i m \varphi}, \\
(m=0, \pm 1, \pm 2 \cdots) .
\end{aligned}
$$

By the method of separation of variables, we are able to divide Eq. (9) into two parts. One is the radial part $R(r)$ that is represented as

$$
\frac{\partial^{2} R(r)}{\partial r^{2}}+\frac{2}{r} \frac{\partial R(r)}{\partial r}+\left(\frac{2 \mu_{0} E_{n}}{\hbar^{2}}-\frac{c}{r^{2}}+\frac{2 \mu_{0} Z_{0}}{\hbar^{2} r}\right) R(r)=0
$$

and the other is the polar angle equation of $\Theta(\theta)$ which is of the form

$$
\frac{\partial^{2} \Theta(\theta)}{\partial \theta^{2}}+\cot \theta \frac{\partial \Theta(\theta)}{\partial \theta}+\left(c-\frac{m^{2}}{\sin ^{2} \theta}-\frac{f(\theta)}{\hbar^{2}}\right) \Theta(\theta)=0 .
$$

After we first solve the radial equation in the next section, the equation of the polar angle will also be solved.

\section{EIGENVALUES AND EIGENFUNCTIONS OF THE RADIAL EQUATION}

The NU method, which is a powerful tool for evaluating generalized second-order linear differential equations in terms of special orthogonal functions, is useful for solving either Eq. (11) or Eq. (12). Let us first find the bound-state solutions of Eq. (11) which is the radial equation. After a minor re-arrangement, Eq. (11) can be written as

$$
R^{\prime \prime}(r)+\frac{2}{r} R^{\prime}(r)+\left[\frac{-\kappa_{n}^{2} r^{2}-\varepsilon^{2} r-l(l+1)}{r^{2}}\right] R(r)=0,
$$

where

$$
\frac{2 \mu_{0} E_{n}}{\hbar^{2}}=-\kappa_{n}^{2}, \quad \frac{2 \mu_{0} Z_{0}}{\hbar^{2}}=-\varepsilon^{2}, \quad c=l(l+1) .
$$

Here, $E_{n}<0$, which means that the system is bounded.

By applying the same method of Ref. [9] when solving Eq. (13), which is based on the NU method, we easily derive the radial wave function such that

$$
\begin{aligned}
& R_{n l}(r)=\left(\frac{\Gamma(n+1)}{2(n+l+1) \Gamma(n+2 l+2)}\right)^{1 / 2} \\
& \quad \times\left(\frac{2 \mu_{0} Z_{0}}{\hbar^{2}(n+l+1)}\right)^{3 / 2}\left(\frac{2 \mu_{0} Z_{0} r}{\hbar^{2}(n+l+1)}\right)^{l} \\
& \quad \times \exp \left(\frac{-\mu_{0} Z_{0}}{\hbar^{2}(n+l+1)} r\right) L_{n}^{2 l+1}\left(\frac{2 \mu_{0} Z_{0}}{\hbar^{2}(n+l+1)} r\right) .
\end{aligned}
$$

These are the bound-state solutions [31], and one can easily check that these equations vanish at $r=0$.

\section{EIGENVALUES AND EIGENFUCTIONS OF THE POLAR ANGLE EQUATION}

Let us take $f(\theta)$ so that the relevant potential becomes a kind of Makarov potential [32], which is a double ring- 
shaped generalized non-central potential:

$$
\frac{f(\theta)}{r^{2}}=\frac{1}{r^{2}}\left(\frac{a}{\sin ^{2} \theta}+\frac{b \cos \theta}{\sin ^{2} \theta}\right),
$$

where $a$ and $b$ are constants that are allowed to be real and positive. We assume that the variation of $\theta$ from the equilibrium angle is sufficiently small in this potential. If we consider the case that $a=1$ and $b=-0.5$ as an example, the equilibrium angle is $\theta_{0}=1.2995$, where the derivative of $f(\theta)$ with respect to $\theta$ results in zero: $f^{\prime}(\theta)=0$.

We solve Eq. (12) in order to obtain spherical harmonics. If we change the variable as $\cos \theta=h$, Eq. (12) can be rewritten as

$$
\frac{d^{2} \Theta(h)}{d h^{2}}-\frac{2 h}{1-h^{2}} \frac{d \Theta(h)}{d h}+\left(\frac{c\left(1-h^{2}\right)-m^{2}-\frac{(a+b h)}{\hbar^{2}}}{\left(1-h^{2}\right)^{2}}\right)
$$

Let us use the NU method [9,15-17], which is useful for solving the solution of this equation. Usually, the NU method starts from the following equation [9]:

$$
\Theta^{\prime \prime}(h)+\frac{\tilde{\tau}(h)}{\sigma(h)} \Theta^{\prime}(h)+\frac{\tilde{\sigma}(h)}{\sigma^{2}(h)} \Theta(h)=0,
$$

$$
\Pi= \pm\left\{\begin{array}{l}
\left(\frac{m^{2}+\left(a / \hbar^{2}\right)+u}{2}\right)^{1 / 2} h+\left(\frac{m^{2}+\left(a / \hbar^{2}\right)-u}{2}\right)^{1 / 2}, \\
\left(\frac{m^{2}+\left(a / \hbar^{2}\right)-u}{2}\right)^{1 / 2} h+\left(\frac{m^{2}+\left(a / \hbar^{2}\right)+u}{2}\right)^{1 / 2}
\end{array}\right.
$$

where the derivative of $\tau$ is negative.

By representing $\Theta_{n^{\prime}}(h)$ in the form

$$
\Theta_{n^{\prime}}(h)=v_{n^{\prime}}(h) y_{n^{\prime}}(h),
$$

where $v_{n^{\prime}}(h)$ is a function of $h$ that is chosen adequately, we see that Eq. (18) reduces to a hypergeometric-type equation as [33]:

$$
\sigma(h) y_{n^{\prime}}^{\prime \prime}+\tau(h) y_{n^{\prime}}^{\prime}+\lambda y_{n^{\prime}}=0
$$

where $\tau(h)$ is a function of $h$, which is of the form $\tau(h)=$ $\tilde{\tau}(h)+2 \Pi(h)$, and $\lambda$ is a constant that is given by $\lambda=$ $k+\Pi^{\prime}$. Hence, from a minor evaluation, we have where various functions of $h, \tilde{\tau}(h), \sigma(h)$, and $\tilde{\sigma}(h)$ should be determined depending on the parameters of particular systems. In this case, by comparing Eqs. (17) and (18), we easily have

$$
\begin{aligned}
& \tilde{\tau}=-2 h, \quad \sigma=1-h^{2}, \\
& \tilde{\sigma}=-c h^{2}-\left(b / \hbar^{2}\right) h+\left(c-m^{2}-a / \hbar^{2}\right) .
\end{aligned}
$$

A weight function $\Pi$ introduced in Ref. [9] is useful for further discussion. This can be derived by substituting the functions of Eq. (19) in Eq. (A8) of Ref. [9] as (see Appendix B)

$$
\begin{aligned}
\Pi= \pm\left[(c-k) h^{2}+\right. & \left(b / \hbar^{2}\right) h-\left(c-m^{2}\right. \\
& \left.\left.-\left(a / \hbar^{2}\right)-k\right)\right]^{1 / 2} .
\end{aligned}
$$

Because the expression in the square root of this equation can be represented as a square of a polynomial, we can identify the mathematically allowed values of $\Pi$, such that

$$
\left.\begin{array}{l}
\text { for } k=\frac{2 c-m^{2}-\left(a / \hbar^{2}\right)-u}{2} \\
\text { for } k=\frac{2 c-m^{2}-\left(a / \hbar^{2}\right)+u}{2}
\end{array}\right\}
$$

$$
\lambda=\frac{2 c-\left(m^{2}+a / \hbar^{2}\right)}{2}-\frac{u}{2}-\left(\frac{m^{2}+\left(a / \hbar^{2}\right)+u}{2}\right)^{1 / 2}
$$

On the other hand, a direct calculation of $\lambda$ from the method used in Ref. [9] yields $\lambda_{n^{\prime}}=-n^{\prime} \tau^{\prime}-\frac{n(n-1)}{2} \sigma^{\prime \prime}$. In this case, the expansion of $\lambda$ becomes

$$
\lambda_{n^{\prime}}=2 n^{\prime}\left[1+\left(\frac{m^{2}+\left(a / \hbar^{2}\right)+u}{2}\right)^{1 / 2}\right]+n^{\prime}\left(n^{\prime}-1\right)
$$

By combining the two equations, Eqs. (25) and (26), we see that

$$
\begin{array}{r}
\left(2 n^{\prime}+1\right)\left(\frac{m^{2}+\left(a / \hbar^{2}\right)+u}{2}\right)^{1 / 2}+\frac{u-\left(m^{2}+a / \hbar^{2}\right)}{2} \\
+n^{\prime}\left(n^{\prime}+1\right)=c-\left(m^{2}+a / \hbar^{2}\right) .
\end{array}
$$


Now from $c=l(l+1)$, we have

$$
l=n^{\prime}+\left(\frac{m^{2}+\left(a / \hbar^{2}\right)+\left(m^{2}+\left(a / \hbar^{2}\right)-\left(b / \hbar^{2}\right)^{2}\right)^{1 / 2}}{2}\right)^{1 / 2} .
$$

By substituting Eq. (28) in a solution of an eigenvlaue equation of the radial part that is given in Eq. (22) of Ref. [9] (see Appendix C), we have the eigenvalues as

$$
E_{n n^{\prime} m}=-\frac{\mu_{0} Z_{0}^{2}}{2 \hbar^{2}} \frac{1}{\left[n+n^{\prime}+\left(\frac{m^{2}+\left(a / \hbar^{2}\right)+\left[\left(m^{2}+a / \hbar^{2}\right)^{2}-\left(b / \hbar^{2}\right)^{2}\right]^{1 / 2}}{2}\right)^{1 / 2}+1\right]^{2}} .
$$

The wave function for the polar angle, given in Eq. (12), is also obtained from the use of $\sigma$ and $\Pi$ on the basis of the NU method. The required functions needed for unfolding the corresponding theory of $\mathrm{NU}$ for this case are as follows (see Appendix A of Ref. [9]):

$$
\begin{gathered}
v(h)=(1-h)^{\left(m_{1}+m_{2}\right) / 2}(1+h)^{\left(m_{1}-m_{2}\right) / 2}, \\
\rho(h)=\left(1-h^{2}\right)^{m_{1}}\left(\frac{1+h}{1-h}\right)^{-m_{2}}, \\
y_{n^{\prime}}(h)=A_{n^{\prime}}(1-h)^{-\left(m_{1}+m_{2}\right)}(1+h)^{-\left(m_{1}-m_{2}\right)} \\
\times \frac{d^{n^{\prime}}}{d h^{n^{\prime}}}\left[(1+h)^{n^{\prime}+m_{1}-m_{2}}(1-h)^{n^{\prime}+m_{1}+m_{2}}\right],
\end{gathered}
$$

where $m_{1}$ and $m_{2}$ are represented as $m_{1}=$ $\left\{\left[m^{2}+\left(a / \hbar^{2}\right)+u\right] / 2\right\}^{1 / 2}$ and $m_{2}=\left\{\left[m^{2}+\left(a / \hbar^{2}\right)\right.\right.$ $-u] / 2\}^{1 / 2}$. Here, $\rho(h)$ is a weight function. For more details on $\rho(h)$, one can refer to Appendix D.

Now, the exact polynomial $y_{n^{\prime}}(h)$ is expressed by using the Jacobi polynomials $P_{n^{\prime}}^{\left(m_{1}+m_{2}, m_{1}-m_{2}\right)}(h)$. If we insert Eqs. (30)-(32) in Eq. (23), the wave functions for the polar angle part can be written as

$$
\begin{array}{r}
\Theta_{n^{\prime}}(\theta)=C_{n^{\prime}}(1-\cos \theta)^{\left(m_{1}+m_{2}\right) / 2}(1+\cos \theta)^{\left(m_{1}-m_{2}\right) / 2} \\
\times P_{n^{\prime}}^{\left(m_{1}+m_{2}, m_{1}-m_{2}\right)}(\cos \theta),
\end{array}
$$

where $C_{n^{\prime}}$ is a normalization factor. $C_{n^{\prime}}$ may be determined from $\int_{-1}^{+1} \Theta_{n}^{*}(h) \Theta_{n^{\prime}}(h) d h=1$, and the result is

$$
C_{n^{\prime}}=\left(\frac{\left(2 n^{\prime}+2 m_{1}+1\right) \Gamma\left(n^{\prime}+1\right) \Gamma\left(n^{\prime}+2 m_{1}+1\right)}{2^{2 m_{1}+1} \Gamma\left(n^{\prime}+m_{1}+m_{2}+1\right) \Gamma\left(n^{\prime}+m_{1}-m_{2}+1\right)}\right)^{1 / 2} .
$$

Then, the eigenfunctions of the invariant operator in the transformed system become

$$
\begin{aligned}
\Phi_{n n^{\prime} m}(r, \theta, \varphi)= & {\left[\frac{\left(2 n^{\prime}+2 m_{1}+1\right) \Gamma\left(n^{\prime}+1\right) \Gamma\left(n^{\prime}+2 m_{1}+1\right)}{2^{2 m_{1}+2} \pi \Gamma\left(n^{\prime}+m_{1}+m_{2}+1\right) \Gamma\left(n^{\prime}+m_{1}-m_{2}+1\right)}\right]^{1 / 2} } \\
& \times\left(\frac{\Gamma(n+1)}{2(n+l+1) \Gamma(n+2 l+2)}\right)^{1 / 2}\left(\frac{2 \mu_{0} Z_{0}}{\hbar^{2}(n+l+1)}\right)^{3 / 2}\left(\frac{2 \mu_{0} Z_{0} r}{\hbar^{2}(n+l+1)}\right)^{l} \\
& \times \exp \left(\frac{-\mu_{0} Z_{0}}{\hbar^{2}(n+l+1)} r\right) L_{n}^{2 l+1}\left(\frac{2 \mu_{0} Z_{0}}{\hbar^{2}(n+l+1)} r\right) \exp (i m \varphi) \\
& \times(1-\cos \theta)^{\left(m_{1}+m_{2}\right) / 2}(1+\cos \theta)^{\left(m_{1}-m_{2}\right) / 2} P_{n^{\prime}}^{\left(m_{1}+m_{2}, m_{1}-m_{2}\right)}(\cos \theta) .
\end{aligned}
$$

From the inverse unitary transformation, the eigenstates of the invariant operator in the original system are obtained as

$$
\begin{aligned}
\phi_{n n^{\prime} m}(r, \theta, \varphi, t)= & U^{-1} \Phi_{n n^{\prime} m}(r, \theta, \varphi) \\
= & \left(\frac{\left(2 n^{\prime}+2 m_{1}+1\right) \Gamma\left(n^{\prime}+1\right) \Gamma\left(n^{\prime}+2 m_{1}+1\right)}{2^{2 m_{1}+2} \pi \Gamma\left(n^{\prime}+m_{1}+m_{2}+1\right) \Gamma\left(n^{\prime}+m_{1}-m_{2}+1\right)}\right)^{1 / 2}\left(\frac{\Gamma(n+1)}{2(n+l+1) \Gamma(n+2 l+2)}\right)^{1 / 2} \\
& \times\left(\frac{2 \mu_{0} Z_{0}}{\hbar^{2}(n+l+1)}\right)^{1 / 2}\left(\frac{2 \mu_{0} Z_{0}}{\hbar^{2}\left(1-k t-\frac{1}{2} k^{2} t^{2}\right)(n+l+1)}\right)^{l+1} r^{l} \exp \left(\frac{i \mu_{0} k}{2 \hbar\left(1-k t-\frac{1}{2} k^{2} t^{2}\right)} r^{2}\right) \\
& \times \exp \left(\frac{-2 \mu_{0} Z_{0}}{\hbar^{2}\left(1-k t-\frac{1}{2} k^{2} t^{2}\right)(n+l+1)} r\right) L_{n}^{2 l+1}\left(\frac{2 \mu_{0} Z_{0}}{\hbar^{2}\left(1-k t-\frac{1}{2} k^{2} t^{2}\right)(n+l+1)} r\right) \\
& \times(1-\cos \theta)^{\left(m_{1}+m_{2}\right) / 2}(1+\cos \theta)^{\left(m_{1}-m_{2}\right) / 2} P_{n^{\prime}}^{\left(m_{1}+m_{2}, m_{1}-m_{2}\right)}(\cos \theta) \exp (i m \varphi) .
\end{aligned}
$$


Now, we need to determine the phases of the wave functions. For this purpose, let us consider the equation for the phases $\theta_{n n^{\prime} m}(t)$, that is [9]

$$
\hbar \frac{d}{d t} \theta_{n n^{\prime} m}(t)=\left\langle\phi_{n n^{\prime} m}(t)\left|\left(i \hbar \frac{\partial}{\partial t}-H\right)\right| \phi_{n n^{\prime} m}(t)\right\rangle
$$

Through a unitary transformation for this equation, we have

$$
\hbar \frac{d}{d t} \theta_{n n^{\prime} m}(t)=\left\langle\Phi_{n n^{\prime} m}(r, \theta, \varphi)\left|-\frac{1+k t}{\left(1-k t-\frac{1}{2} k^{2} t^{2}\right)^{2}} I_{0}\right| \Phi_{n n^{\prime} m}(r, \theta, \varphi)\right\rangle .
$$

Now, we easily solve this equation and find

$$
\theta_{n n^{\prime} m}(t)=\frac{\mu_{0} Z_{0}^{2}}{2 \hbar^{3}} \frac{1}{k\left(1-k t-\frac{1}{2} k^{2} t^{2}\right)}\left[n+n^{\prime}+1+\left(\frac{m^{2}+\left(a / \hbar^{2}\right)+\left[\left(m^{2}+a / \hbar^{2}\right)^{2}-\left(b / \hbar^{2}\right)^{2}\right]^{1 / 2}}{2}\right)^{1 / 2}\right]^{-2}
$$

According to the invariant operator theory, the wave functions of the system which is time-dependent are given by [34] $\psi_{n n^{\prime} m}(t)=e^{i \theta_{n n^{\prime} m}(t)} \phi_{n n^{\prime} m}(t)$. Thus, in terms of Eqs. (36) and (39), the full wave functions in the original system can be represented as

$$
\begin{aligned}
\psi_{n n^{\prime} m}(r, \theta, \varphi, t)= & \left(\frac{\left(2 n^{\prime}+2 m_{1}+1\right) \Gamma\left(n^{\prime}+1\right) \Gamma\left(n^{\prime}+2 m_{1}+1\right)}{2^{2 m_{1}+2} \pi \Gamma\left(n^{\prime}+m_{1}+m_{2}+1\right) \Gamma\left(n^{\prime}+m_{1}-m_{2}+1\right)}\right)^{1 / 2}\left(\frac{\Gamma(n+1)}{2(n+l+1) \Gamma(n+2 l+2)}\right)^{1 / 2} \\
& \times\left(\frac{2 \mu_{0} Z_{0}}{\hbar^{2}(n+l+1)}\right)^{1 / 2}\left(\frac{2 \mu_{0} Z_{0}}{\hbar^{2}\left(1-k t-\frac{1}{2} k^{2} t^{2}\right)(n+l+1)}\right)^{l+1} r^{l} \exp \left(\frac{i \mu_{0} k}{2 \hbar\left(1-k t-\frac{1}{2} k^{2} t^{2}\right)} r^{2}\right) \\
& \times \exp \left(\frac{-\mu_{0} Z_{0}}{\hbar^{2}\left(1-k t-\frac{1}{2} k^{2} t^{2}\right)(n+l+1)} r\right) L_{n}^{2 l+1}\left(\frac{2 \mu_{0} Z_{0}}{\hbar^{2}\left(1-k t-\frac{1}{2} k^{2} t^{2}\right)(n+l+1)}\right) \\
& \times(1-\cos \theta)^{\left(m_{1}+m_{2}\right) / 2}(1+\cos \theta)^{\left(m_{1}-m_{2}\right) / 2} P_{n^{\prime}}^{\left(m_{1}+m_{2}, m_{1}-m_{2}\right)}(\cos \theta) \\
& \times \exp \left(i \theta_{n n^{\prime} m}(t)+i m \varphi\right) .
\end{aligned}
$$

The wave functions given here are very useful for investigating the quantum properties of the system. The expectation values of the canonical variables, quantum observables, and other quantum variables can be evaluated by means of these wave functions. Also, quantum fluctuations, uncertainty relations, correlations between canonical variables, and phase properties of eigenstates can be studied through the use of the wave functions. These analytical wave functions are better than the wave functions obtained from numerical or perturbation methods. Numerical solutions of the wave functions are somewhat not flexible. Hence, a set of data for the wave functions obtained at a certain time $t$ by using a numerical method cannot be used as the data at a different time. However, from the analytical wave functions, we can easily identify the whole spectrum of eigenstates and eigenvalues.

\section{CONCLUSION}

Exact wave functions for a TDHS that have a timevariable mass subjected to time-dependent singular potentials in 3 dimensions have been investigated. A timedependent inverse quadratic potential and a Coulomb- like potential were considered as singular potentials. From the Hamiltonian of the system, an invariant operator which is useful for studying the quantum properties of the system has been constructed. Because the invariant operator is somewhat complicated, as shown in Eq. (3), we transformed it to a simple form by means of a unitary operator. The invariant operator in the transformed system does not depend on time, and we easily obtained its eigenstates by using the NU method. Analytical solutions for both the radial equation, Eq. (13), and the polar angle equation, Eq. (17), are exactly derived by using the $\mathrm{NU}$ method, leading to recognition of the complete eigenstates of $I_{0}$. From the inverse transformation of the eigenstates represented in the transformed system to those of the original system, complete eigenstates of the invariant operator $I$ are identified. We have supposed, according to the theory of Lewis and Riesenfeld [34], that the full wave functions of the system are represented in terms of $\phi_{n}$ and that the corresponding phases of the wave functions are derived with the aid of the Schrödinger equation. From this procedure, the full wave functions are identified.

We considered the double ring-shaped generalized noncentral potential in this work, which is a kind of Makarov 
potential [32]. To now, much research has been done concerning the double ring-shaped generalized non-central potential defined in Eq. (16) $[14,33,35,36]$. However, the quantum problem of this potential system with timedependent parameters has not been reported yet as far as we know. Perhaps, this is due to the difficulty of mathematical procedures for the complicated system in the time-dependent domain.

The complete wave functions obtained in this work are useful for investigating various quantum properties of the system. For instance, they enable us to derive energy eigenstates and to compare them with their classical counterparts. They are also useful for studying quantum characteristics of other quantum observables of the system. The fluctuation of canonical variables, the uncertainty products, probability densities, density operators, and the Wigner distribution functions can be clarified by means of the wave functions.

As a next task for research, a study of the phase properties of the system may be a worthwhile topic. The phases $\theta_{n n^{\prime} m}(t)$ are composed of the usual dynamical phases and geometrical ones. Among them, the geometrical phases have many applications in physical science. For instance, geometrical phases can be applied to the theory of optical spin rotations in quantum dot [37], to the analysis of bonding states of molecules [38], in realizing geometric phase gates in quantum computing [39], and so forth. Indeed, the study of geometrical phases provides a powerful new way for understanding dynamical systems because they play essential and important roles in both non-adiabatic and adiabatic evolutions of quantum systems.

\section{APPENDIX A}

In general, the derivation of Schrödinger solutions for time-varying singular potential systems are very difficult, except for the case in which the form of the singular potential is a special inverse quadratic type coupled to an harmonic oscillator [40]. Still, we do not know how to derive quantum solutions if we choose arbitrary types of time-varying functions for the Hamiltonian given in Eq. (1). However, from our experience, we have confirmed that we can derive quantum solutions of the system if we choose the time-varying factor of $1 / r$ in the Hamiltonian to be the form shown in Eq. (1).

\section{APPENDIX B}

The exact formula of Eq. (A8) of Ref. [9] is given by

$$
\begin{aligned}
\Pi(h)= & \frac{\sigma^{\prime}(h)-\tilde{\tau}(h)}{2} \\
& \pm \sqrt{\left(\frac{\sigma^{\prime}(h)-\tilde{\tau}(h)}{2}\right)^{2}-\tilde{\sigma}(h)+k \sigma(h) .}
\end{aligned}
$$

\section{APPENDIX C}

In terms of the notations of this paper, the formula of Eq. (22) of Ref. [9] is given by

$$
E_{n}=-\frac{\mu_{0} Z_{0}^{2}}{2 \hbar^{2}} \frac{1}{(n+l+1)^{2}}, n=0,1,2, \cdots .
$$

\section{APPENDIX D}

The weight function $\rho(h)$ given in Eq. (31) is necessary for unfolding the NU theory. In general, it is determined from $[9,41]$

$$
[\sigma(h) \rho(h)]^{\prime}=\tau(h) \rho(h) .
$$

\section{ACKNOWLEDGMENTS}

The work of JRC was supported by the Basic Science Research Program of the year 2015 through the National Research Foundation of Korea (NRF) funded by the Ministry of Education (Grant No.: NRF2013R1A1A2062907).

\section{REFERENCES}

[1] H. R. Lewis, Jr., Phys. Rev. Lett. 18, 510 (1967).

[2] J. R. Choi and S. Zhang, J. Phys. A: Math. Gen. 35, 2845 (2002).

[3] J-Y. Ji and J. K. Kim, Phys. Rev. A 53, 703 (1996).

[4] J. R. Choi, Phys. Scr. 68, 36 (2003).

[5] M. Molski, Eur. Phys. J. D 40, 411 (2006).

[6] J. R. Choi and I. H. Nahm, Int. J. Theor. Phys. 46, 1 (2007).

[7] S. Menouar and J. R. Choi, Ann. Phys. 353, 307 (2015).

[8] J. R. Choi, Phys. Dark Univ. 2, 41 (2013).

[9] S. Menouar and J. R. Choi, J. Korean Phys. Soc. 67 1127 (2015).

[10] H. X. Quan, L. Guang, W. Z. Min, N. L. Bin and M. Yan, Commun. Theor. Phys. 53, 242 (2010).

[11] Z. M. Cang and W. Z. Bang, Chinese Phys. 16, 1863 (2007).

[12] S. Flügge, Practical Quantum Mechanics I (Springer, Heidelberg, New York, 1971).

[13] Y. Aharonov and D. Bohm, Phys. Rev. 115, 485 (1959).

[14] B. Gönül and I. Zorba, Phys. Lett. A 269, 83 (2000).

[15] J. R. Choi and J. Y. Oh, Int. J. Theor. Phys. 46, 2591 (2007).

[16] J. R. Choi, Int. J. Theor. Phys. 42, 853 (2003).

[17] S. Menouar, M. Maamache, H. Bekkar and J. R. Choi, J. Korean Phys. Soc. 58, 154 (2011).

[18] A. Schulze-Halberg, Phys. Res. Intern. 2008, 589269 (2008).

[19] M. Vubangsi, M. Tchoffo and L. C. Fai, African Rev. Phys. 8, 341 (2013). 
[20] Y. Ben-Aryeh, arXiv:gr-qc/0807.4670 (2008).

[21] J. R. Choi and J. H. Gweon, J. Korean Phys. Soc. 43, 17 (2003).

[22] C. I. Um, J. R. Choi and K. H. Yeon, J. Korean Phys. Soc. 38, 452 (2001).

[23] H. Moya-Cessa and M. Fernández Guasti, Revista Mexicana de Física 53, 42 (2007).

[24] R. K. Colegrave and M. S. Abdalla, J. Phys. A: Math. Gen. 14, 2269 (1981).

[25] C-I. Um, I-H. Kim and K-H. Yeon, in Current Topics in Physics, editted by Y. M. Cho, J. B. Hong and C. N. Yang (World Scientific, Singapore, 1996), vol. 1, p. 510, Proceedings of the Inauguration Conference of the Asia-Pacific Center for Theoretical Phtsics.

[26] A. V. Zhukov and P. N. Zhukova, J. Phys. A: Math. Gen. 32, 1779 (1999).

[27] C-I. Um, I-H. Kim, K-H. Yeon, T. F George and L. N. Pandey, J. Phys. A Math. Gen. 30, 2545 (1997).

[28] Ö. Yeşiltaş and R. Sever, arxiv:quant-ph/0703034v1 (2007).

[29] C-Y. Chen, C-L. Liu and F-L. Lu, Phys. Lett. A 374, 1346 (2010).

[30] M-C. Zhang and G-Q. Huang-Fu, J. Math. Phys. 52, 053518 (2011).
[31] R. L. Liboff, Introductory Quantum Mechanics, 4th ed. (Addison Wesley, San Fransisco, 2002).

[32] A. A. Makarov, J. A. Smorodinsky, K. H. Valiev and P. Winternitz, Nuovo Cimento A 52, 1061 (1967).

[33] F. Yaşuk, C. Berkdemir and A. Berkdemir, J. Phys. A: Math. Gen 38, 6579 (2005).

[34] H. R. Lewis, Jr. and W. B. Riesenfeld, J. Math. Phys. 10, 1458 (1969).

[35] J. Sadeghi and B. Pourhassan, Elect. J. Theor. Phys. 5, 193 (2008).

[36] X-C. Gao, J. Fu, J. Xu and X. Zou, Phys. Rev. A 59, 55 (1999).

[37] S. E. Economou and T. L. Reinecke, Phys. Rev. Lett. 99, 217401 (2007).

[38] J. Bhattacharjee, S. Narasimhan and U. V. Waghmare, arXiv:cond-mat/0612468 (2006).

[39] A. Lemmer, A. Bermudez and M. B. Plenio, New J. Phys. 15, 083001 (2013).

[40] J. R. Choi and B. H. Gweon, Int. J. Mod. Phys. B 16, 4733 (2002).

[41] A. F. Nikiforov and V. B. Uvarov, Special Functions of Mathematical Physics (Birkhäuser Verlag Basel, Germany, 1988). 